\title{
Conocimiento de los profesionales de enfermería sobre Diabetes Mellitus Tipo 2, Villavicencio, Meta, 2010
}

\author{
Nurses' knowledge on Diabetes Mellitus Type 2, \\ Villavicencio, Meta, 2010
}

Conhecimento dos profissionais da enfermagem
sobre a Diabetes Mellitus Tipo 2, Villavicencio, Meta, 2012

Luz H. Aponte-Garzón ${ }^{1 *}$; Ruth F. Hernández-Páez ${ }^{2}$

* Enfermeras, MSc,
* Grupo de Investigación de Cuidado, Facultad de Ciencias de la Salud, Universidad de los Llanos, Villavicencio, Colombia.
Email: grupocuidado@unillanos.edu.co

Recibido: noviembre 22 de 2011. Aceptado: mayo 10 de 2012

\begin{abstract}
Resumen
Con el propósito de identificar las necesidades de conocimiento en diabetes mellitus tipo 2 de los enfermeros del área clínica y comunitaria del municipio de Villavicencio (Meta) se desarrolló una investigación de diseño observacional, descriptivo transversal. Los enfermeros fueron seleccionados del área clínica y comunitaria de diferentes instituciones de salud por medio de muestreo por conveniencia $(n=30)$ y se les aplicó un instrumento de valoración de conocimientos. La valoración de conocimientos reporta limitaciones importantes en los conocimientos sobre el tema de diabetes mellitus tipo 2. La relación entre nivel de conocimientos y tiempo de experiencia laboral fue examinada por medio de prueba de Fisher para cada una de las áreas clínica y comunitaria ( $p=0,46$ y 0,60 respectivamente). Se desarrolló un componente cualitativo para conocer las características de la educación que los profesionales de enfermería impartían a las personas con diabetes y las limitaciones percibidas por los profesionales en el cuidado, este componente se adelantó por medio de entrevistas semi-estructuradas $(n=10)$ y se realizó análisis de contenido por medio de la identificación de segmentos con patrones específicos y codificación para identificación de categorías de análisis. Las categorías identificadas fueron la sobrecarga laboral, el desempeño de funciones no profesionales, la delegación de funciones de cuidado y educación a personal no profesional, la necesidad de educación continuada, los recursos insuficientes en los programas de promoción y prevención, y la necesidad de apoyo técnico y de trabajo interdisciplinario, entre otras.
\end{abstract}

Palabras clave: Diabetes Mellitus Tipo 2, Cuidado de Enfermería, Enfermedad Crónica.

\begin{abstract}
In order to identify the clinical and community nurses' knowledge level about diabetes type 2 an observational, descriptive, cross-sectional was developed. The study was carried out in Villavicencio (Meta), participants were selected using a convenience sampling $(n=30)$ A knowledge assessment instrument designed for this study was applied. Results showed knowledge limitations in the vast majority of nurses, relation between years of experience in clinical and community settings and level of knowledge was explored using Fisher test obtaining $p$ values $=0,46$ and 0,60 respectively. A qualitative
\end{abstract}


component was carried out using semi-structured interviews $(n=10)$, with content analysis and identification of relevant analysis categories. Identified categories included work overload, performance of non-professional duties, delegation of care to non professional nursing personnel, lack of continuing education, need for participation of other health professionals for more comprehensive care.

Key words: Diabetes Mellitus Type 2, Nursing Care, Chronic Diseases.

\begin{abstract}
Resumo
Com o identificar as necessidades do conhecimento em diabetes mellitus tipo 2 dos profissionais da enfermagem da área clinica e comunitária de Villavicencio (Meta) foi realizada esta pesquisa com um delineamento de observação, descritivo transversal. Os profissionais da enfermagem foram selecionados da área clinica e comunitária em diferentes instituições de saúde mediante amostragem por conveniência $(n=30)$ aplicando um instrumento para valoração de conhecimentos. A valoração de conhecimentos falências nos mesmos sobre a diabetes mellitus tipo 2 . A relação entre nível de conhecimentos e tempo de experiência laboral foi analisada pela prova de Fisherpara cada área clinica e comunitária ( $p=0,46$ e 0,60 respetivamente). Desenvolveu-se um componente qualitativo para conhecer as características da educação que os profissionaisda enfermagem oferecem ás pessoas com diabetes e as limitações percebidas pelos profissionais na atenção dos pacientes, para este componente foram aplicadas entrevista semi-estruturadas $(n=10)$ e o analise de conteúdo foi realizado pela identificação de segmentos com padrões específicos e codificados para as categorias de analises. As categorias identificadas foram sobrecarga laboral, desempenho de funções não laborais, delegação de funções de atenção e educação a pessoal não profissional, a necessidade de educação continuada, recursos escassos nos programas de promoção e prevenção e a necessidade de apoio técnico do trabalho interdisciplinar entre outras.
\end{abstract}

Palabras chave: Diabetes Mellitus Tipo 2, Cuidado de Enfermagem, doença crônica.

\section{Introducción}

La Diabetes Mellitus tipo 2 (DM2) es una de las enfermedades crónicas más prevalentes a nivel mundial y el incremento en el número de casos cada año es sostenido, el $63 \%$ del total de muertes anuales, correspondientes a 36 millones de muertes en el mundo para 2008 se asociaron a la presencia de enfermedades crónicas, entre las que se incluye la diabetes. (OMS, 2010) En el continente americano es una enfermedad frecuente entre los adultos, que afecta de manera importante la calidad de vida de los enfermos y de sus familias (OPS, 2009a). Los retos que esta enfermedad impone al enfermo y a sus familias son múltiples y sus secuelas, en la mayoría de los casos, severas. La DM2 es una enfermedad de muy alto costo económico para el sistema de salud y para la sociedad. En Estados Unidos, los costos anuales han sido estimados en $\$ 135$ billones de dólares, la mayor parte relacionado con los costos por hospitalización, servicios de rehabilitación, diálisis y pérdida de productividad y empleo relacionados con discapacidad asociada a la diabetes (Weiler y Crist, 2007).

Según los datos reportados por el PRB, Latinoamérica tiene 580 millones de habitantes (PRB, 2009), de los cuales por lo menos 20 millones padecen DM2 (OPS, 2009b). Por esta razón, los enfermeros deben estar preparados para ofrecer un cuidado integral que in- volucre las diferentes esferas de desarrollo personal y familiar, por lo que requieren contar con una formación específica en el cuidado de este tipo de pacientes, que abarque toda su complejidad.

De hecho, los requerimientos de los pacientes diabéticos han sido por décadas un reto en el cuidado que enfermería ofrece tanto a nivel institucional como comunitario y familiar. En la actualidad, con la tendencia de globalización, y adopción de estilos de vida poco saludables, sumados al aumento de la expectativa de vida de la población general se ha hecho evidente el incremento de los casos de DM2 en la población mundial (Bradbury-Jones, 2009). Ante esta situación, el personal de enfermería debe contar con los conocimientos y formación para cuidar de los pacientes y familias con miras a obtener y mantener el control metabólico. El enfermo diabético y sus familias, esperan y deben recibir un cuidado de enfermería de calidad, que incluya una educación que les permita realizar las acciones de autocuidado apropiadas para el manejo de esta enfermedad, de manera que se mantenga, al menor nivel posible la probabilidad de desarrollar secuelas y daños en órgano blanco (Hill, 2009; Adams, 2009; Beto y Nicholas, 2009).

A pesar de que el personal de enfermería reconoce la importancia de la diabetes como un problema de gran magnitud, las investigaciones señalan que la 
formación de enfermeros de servicio clínico y comunitario debe ser ampliada y profundizada con el objeto de ofrecer el mejor cuidado posible al paciente diabético y sus familias (Hicks, 2009; García, 2007).

El propósito del presente estudio fue el de determinar las necesidades de conocimiento en DM2 de profesionales de enfermería del área clínica y comunitaria del municipio de Villavicencio, Meta por medio de la valoración de los conocimientos que los profesionales de enfermería tienen para el cuidado de la persona con DM2 y la determinación de las formas como se da educación sobre diabetes a las personas, familia y comunidad usuarias del servicio de enfermería en Villavicencio.

\section{Materiales y métodos}

El trabajo de investigación desarrollado tuvo un diseño de tipo observacional, descriptivo transversal. El componente cualitativo se adelantó por medio del análisis de contenido y la correspondiente identificación de categorías de análisis que den cuenta de los aspectos más relevantes de la experiencia de los profesionales cuando dan educación en salud a las personas con DM2.

El universo estuvo conformado por los enfermeros del área clínica y comunitaria que cuidan a personas con DM2 en el municipio de Villavicencio. La muestra estuvo conformada por enfermeros del área clínica y comunitaria $(n=30)$ que cuidan a personas adultas con DM2 y que cumplieron con los criterios de inclusión. En el área clínica se incluyeron las tres instituciones del servicio clínico-hospitalario de mayor cobertura de población. Para el área comunitaria se seleccionaron una institución pública y una privada que ofrecían servicios del programa de atención a personas con enfermedades crónicas.

Para los enfermeros del área clínica los criterios de inclusión fueron que laboraran en los servicios de Medicina Interna, Quirúrgicos y Ortopedia y Servicios de Urgencias de adultos, y tuvieran una experiencias mínima de seis meses en el cuidado de personas con DM2 en sus sitios de trabajo. Para los enfermeros del área comunitaria se requería que estuvieran vinculados a programas de atención de personas con enfermedades crónicas o servicios de atención de adultos con enfermedades crónicas por lo menos durante seis meses.

La selección de los participantes se hizo por método no probabilístico, de tipo intencional o por conveniencia. La unidad de muestreo es cada profesional de enfermería participante en el estudio. La unidad de análisis es la misma unidad de muestreo.

Para la recolección de información de carácter cuantitativo, se elaboró un instrumento autoadministrable, ad hoc conformado por 30 preguntas de opción múltiple de tipo argumentativo y propositivo sobre aspectos de cuidado de enfermería a personas con DM2, que abarcaban los temas de bases fisiopatológicas, factores de riesgo, diagnóstico, complicaciones agudas y crónicas, tratamiento farmacológico y no farmacológico y medidas de prevención de la enfermedad y de aparición de complicaciones, entre otros aspectos relacionados con el cuidado de enfermería a la personas con DM2. Para la elaboración de este instrumento se tuvo en cuenta la Guía de atención de Diabetes Mellitus tipo 2 del Ministerio de la Protección Social (Ministerio de la Protección Social, 2008) y el material educativo Cartilla de la Diabetes, preparado por la Asociación Colombiana de Diabetes (Asociación Colombiana de Diabetes, 2004).

Este instrumento incluye además la variable de tiempo de experiencia laboral en servicios de hospitalización (áreas de medicina interna y pre y postoperatorio) y urgencias de adultos, y experiencia laboral en el cuidado de adultos en el área de medicina interna o al cuidado de personas adultas con DM2 en el área ambulatoria.

El instrumento se sometió primero a revisión por una enfermera experta en el tema de diabetes mellitus vinculada a una organización del nivel nacional y dedicada a la atención de personas con diabetes con el objeto de examinar la validez de contenido; luego de la revisión y ajuste correspondiente, el instrumento se sometió a una prueba piloto con enfermeros voluntarios que atendían a personas con DM2 y que laboraban en el área clínica y comunitaria de un municipio del departamento del Meta, diferente al municipio de Villavicencio.

Una vez terminada la afinación del instrumento se invitaron a participar a los enfermeros que cumplían con los criterios de inclusión. Los profesionales que aceptaron participar firmaron un consentimiento informado, de conformidad con lo establecido en la Resolución 08430 de 1993 del Ministerio de Salud. Esta investigación se clasificó como Investigación sin riesgo debido a la aplicación de un cuestionario en la que no se realiza una intervención o modificación intencionada de variables biopsicosociales y no se identifica al participante.

La información recolectada con el instrumento cuantitativo se tabuló, graficó y se realizó análisis univaria- 
do descriptivo con el software Excel 2007. Se realizó análisis bivariado con el tiempo de experiencia laboral en años como variable independiente y grado de conocimientos en DM2 como dependiente aplicando el test exacto de Fisher. La selección de la prueba de Fisher se hizo en razón de la utilidad de esta prueba para la valoración de las diferencias entre proporciones o razones obtenidas a partir de resultados de la medición de una variable dependiente nominal constituida en este estudio por la variable área de experiencia y desempeño laboral y de una variable independiente nominal expresada con un conjunto de datos independientes y referida en este estudio como resultado aprobatorio o no aprobatorio de la prueba de conocimientos.

En el instrumento cuantitativo se valoró cada respuesta correcta con 2 puntos y se calificó de acuerdo a la escala: Menor a 34 puntos corresponde a un conocimiento bajo; entre 34 y 47 puntos corresponde a un conocimiento regular, que incluye un valor aprobatorio de 36 puntos, e igual o mayor a 48 puntos corresponde a un conocimiento alto.

Para los aspectos cualitativos del estudio, se desarroIlaron entrevistas semi-estructuradas a enfermeras y enfermeros que habían participado en la prueba escrita. Las entrevistas fueron grabadas previa autorización del participante. En las entrevistas se recolectó información sobre las condiciones en las que se realiza el cuidado de enfermería a las personas con diabetes a su cuidado y la forma como se daba educación a las personas, familia y comunidad. Esta recolección se adelantó hasta obtener saturación de las respuestas $(n=6$ del área clínica y $n=4$ del área comunitaria). Los resultaron de las entrevistas se sometieron a análisis de contenido y determinación y análisis de categorías. Una vez realizadas las transcripciones de las entrevistas, los textos generados fueron revisados para identificación de enunciados significativos que denotaran la presencia y características específicas de los fenómenos bajo estudio. Estos enunciados seleccionados fueron codificados y agrupados en conjuntos de enunciados afines que mostraban patrones, estructuras definidos y conceptos que conectaban los enunciados entre sí, dando lugar a las categorías. Luego de la generación de categorías estas fueron revisadas y analizadas hasta obtener enunciados codificados en las categorías más afines y categorías que reflejaran de manera más comprehensiva el fenómeno bajo estudio identificado.

Como beneficio por participación y una vez finalizado la recolección de información, se ofreció a las enfermeras y enfermeros participantes, información actualizada sobre el cuidado a personas con diagnóstico de DM2.

\section{Resultados}

De los profesionales de enfermería participantes en el estudio que laboran en el área clínica, el 60\% tiene experiencia laboral de 5 años o menos. Este grupo de profesionales tiene un promedio de experiencia laboral en clínica de 6,53 años (DS $\pm 6,27$ ), con valor máximo de 27 años y mínimo de 1,5. La mediana y moda se ubican en 5 años, por lo que este valor fue usado para agrupar por tiempo de experiencia laboral y examinar la relación con desempeño en la prueba de conocimientos sobre DM2. La figura 1 muestra la relación entre la experiencia laboral en el área clínica y experiencia con pacientes adultos del área de medicina interna, allí se muestra gran concordancia entre ambas por lo que se establece que en la práctica profesional, las oportunidades de cuidar a personas con DM2 se dan en forma amplia en su experiencia laboral.

En lo relacionado con los profesionales de enfermería del área comunitaria el promedio de años de experiencia es de 5.46 años (DS \pm 3.38 ), ligeramente inferior a las profesionales del área clínica. Como se observa en la figura 2, el valor máximo y mínimo de años de experiencia laboral en área comunitaria es de 13 y 2 respectivamente. En la experiencia con adultos crónicos, los profesionales de enfermería reportan un promedio de 4.13 años de experiencia laboral (DS \pm 2.35 ) y una experiencia máxima de ocho años y mínimo de un año. Del total de profesionales de enfermería del área comunitaria, un $46.67 \%$ tienen 5 o más años de experiencia en esa área y de estos, el $40 \%$ tienen cinco o más años de experiencia laboral en servicios comunitarios que involucran a adultos crónicos.

En lo relacionado con los resultados de la valoración de los conocimientos que tienen los participantes sobre DM2, la gran mayoría de los puntajes están por debajo del valor de aprobación (28/30), y se ubican alrededor del valor de 25 puntos, con ubicación de la línea de mejor ajuste en escala entre 23 y 31 puntos. La figura 3 presenta la distribución puntual de la valoración de los conocimientos sobre DM2 que tienen los participantes sobre DM2 y la correspondiente curva de mejor ajuste. Se observa que se encuentran por debajo de valores aprobatorios tanto para enfermeros del área clínica como comunitaria por lo que se identifica la existencia de marcadas limitaciones en los conocimientos sobre el tema de DM2 necesarios para dar el cuidado de enfermería y la educación a las personas con esta enfermedad. 


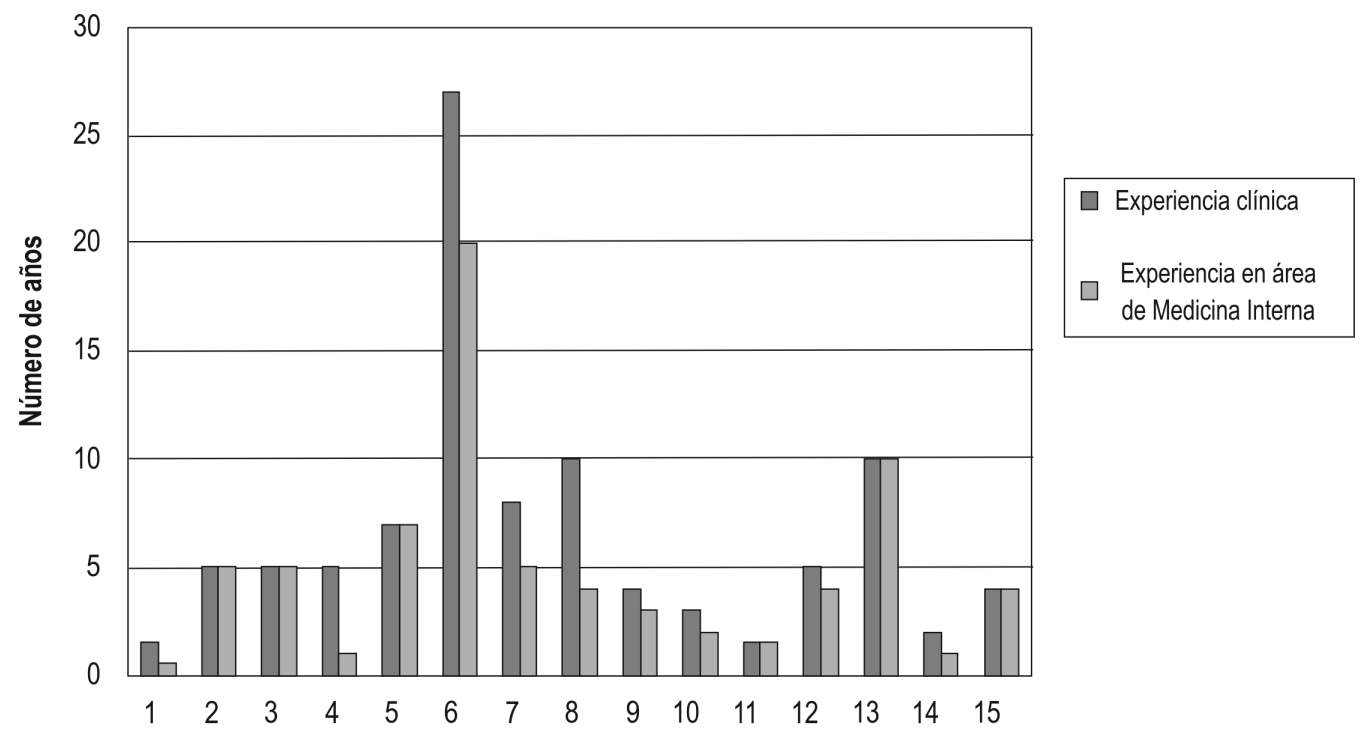

Figura 1. Tiempo de experiencia laboral de los profesionales de enfermería del área clínica, Villavicencio, Meta, 2010.

Al relacionar el tiempo de experiencia laboral en el área clínica y comunitaria y los resultados de la valoración de conocimientos en DM2 de los profesionales de enfermería participantes no se encuentra relación estadísticamente significativa entre nivel de conocimientos y tiempo de experiencia laboral (Ver Tabla 1). Es notorio que la gran mayoría no obtiene nivel de aprobación en la prueba de conocimientos en DM2 $(93,33 \%)$ y que la distribución de profesionales entre las áreas comunitaria y clínica según los años de experiencia laboral es comparable. Es decir, todos los participantes, tienen la oportunidad de dar cuida- do de enfermería a personas con DM2 en su práctica profesional usual.

La Tabla 2 reporta una distribución similar de resultados de la valoración de conocimientos entre los profesionales de enfermería del área clínica y los del área comunitaria. El mayor porcentaje de resultados se encuentra en nivel bajo de conocimientos sobre DM2.

La Tabla 3 muestra que hubo un discreto incremento entre los profesionales de enfermería del área comunitaria de resultados de nivel alto y regular con respec-

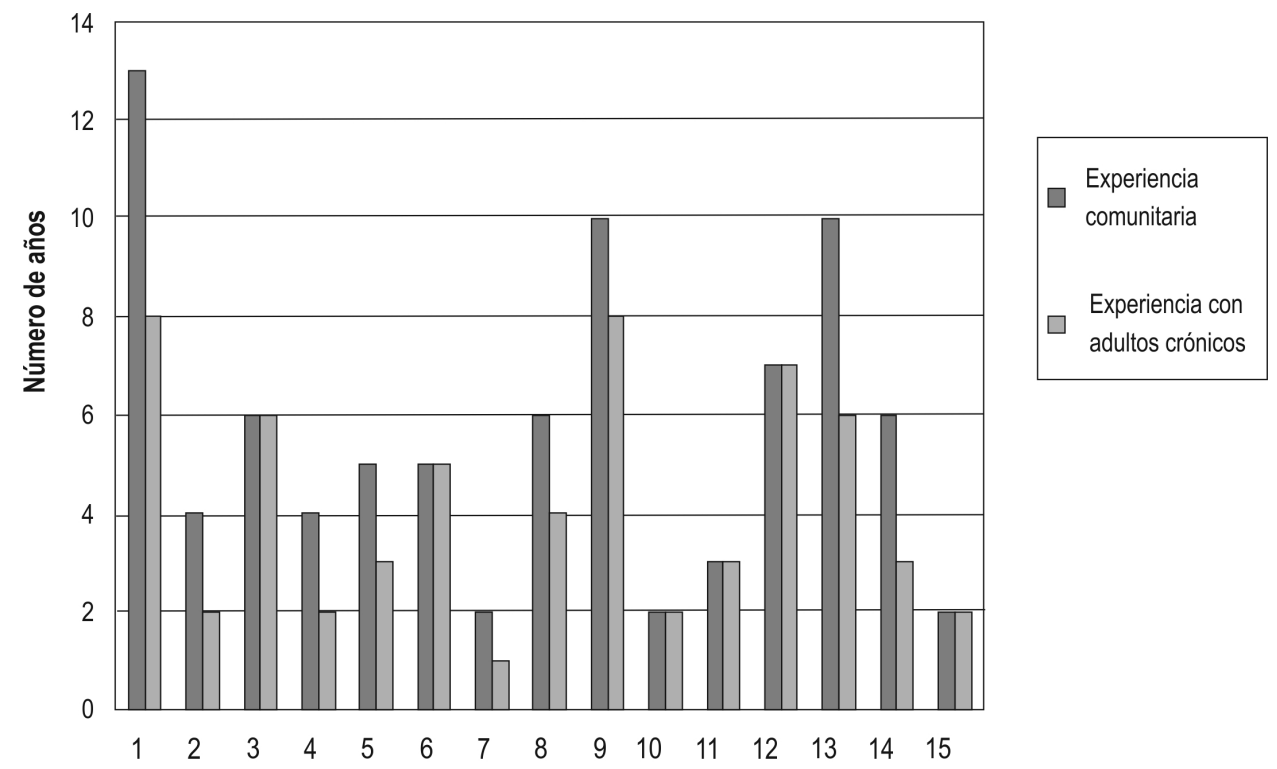

Figura 2. Tiempo de experiencia laboral de los profesionales de enfermería del área comunitaria, Villavicencio, Meta, 2010. 


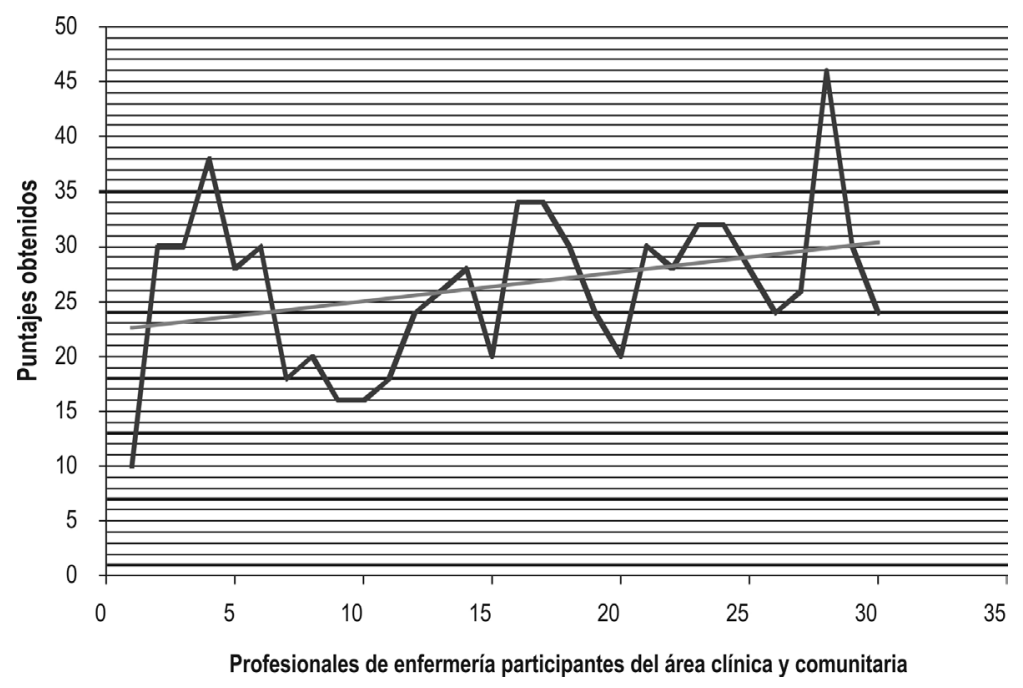

Figura 3. Puntajes obtenidos por los profesionales de enfermería del área clínica y comunitaria en la valoración de conocimientos sobre Diabetes Mellitus tipo 2. Villavicencio, Meta, 2010.

Tabla 1. Relación entre el tiempo de experiencia laboral, área de experiencia y desempeño en la prueba de conocimientos sobre Diabetes Mellitus tipo 2, Villavicencio, Meta, 2010.

\begin{tabular}{l|c|c|c|c}
\hline $\begin{array}{l}\text { Tiempo trabajado } \\
\text { como enfermer@ }\end{array}$ & $\begin{array}{c}\text { Prueba } \\
\text { aprobada }\end{array}$ & $\begin{array}{c}\text { Prueba no } \\
\text { aprobada }\end{array}$ & Total (\%) & P* \\
\hline Comunitaria & $1(6.67)$ & $14(93.33)$ & $15(100)$ & \\
5 años & 0 & $8(100)$ & $8(53.33)$ & 0.46 \\
$>5$ años & $1(14.28)$ & $6(85.72)$ & $7(46.67)$ & \\
Clínica & $1(6.67)$ & $14(93.33)$ & $15(100)$ & \\
5 años & $1(11.12)$ & $8(88.88)$ & $9(60.0)$ & 0.60 \\
$>5$ años & 0 & $6(100)$ & $6(40.0)$ & \\
Total & $2(6.67)$ & $28(93.33)$ & $30(100)$ & \\
\hline
\end{tabular}

* Test exacto de Fisher

to a la proporción de resultados de conocimientos de nivel bajo. Estos conocimientos se refieren a aspectos relacionados con las bases fisiopatológicas, factores de riesgo y medios de diagnóstico. Para los profesionales del área clínica, la respuesta de nivel alto y regular son aún más discretas, por lo que le corresponde un alto porcentaje de resultados (80\%) de nivel bajo.

Las Tablas 4 y 5 muestran similitud en los resultados de la valoración del grado de conocimientos que los profesionales de enfermería participantes tienen sobre mantenimiento del control metabólico y sobre complicaciones de la DM2. Dentro de la valoración de conocimientos sobre el mantenimiento del control metabólico se incluyeron aspectos específicos de tratamiento farma- cológico, recomendaciones en la alimentación, actividad física y ejercicio, etc. En el tema de complicaciones se incluyeron tanto prevención, como detección y cuidado de complicaciones agudas y crónicas de DM2.

Dentro del análisis cualitativo se realizó la identificación de los aspectos comunes que caracterizan la acción de los profesionales de enfermería en las intervenciones educativas a las personas con DM2 tanto en el área clínica como en el área comunitaria. En las entrevistas se indagó sobre las posibilidades y oportunidades para dar educación a las personas con DM2; las limitaciones más significativas para el cuidado de enfermería; y las opciones de mejoramiento para el cuidado de enfermería suministrado. 
Tabla 2. Grado de conocimiento sobre Diabetes Mellitus tipo 2 de los profesionales de enfermería del área clínica comparado con el área comunitaria, Villavicencio, 2010.

\begin{tabular}{l|c|c|c|c|c|c}
\hline \multirow{2}{*}{ Área } & \multicolumn{4}{|c|}{ Grado de conocimiento sobre Diabetes Mellitus Tipo 2 } \\
\cline { 2 - 7 } & \multicolumn{2}{|c|}{ Alto } & \multicolumn{2}{c}{ Regular } & \multicolumn{2}{c}{ Bajo } \\
\cline { 2 - 7 } & $\mathbf{n}$ & $\mathbf{n}$ & $\mathbf{n}$ & $\mathbf{n}$ & $\%$ \\
\hline Clínica & 0 & 0 & 1 & 6.67 & 14 & 93.33 \\
Comunitaria & 0 & 0 & 3 & 20 & 12 & 80 \\
\hline
\end{tabular}

Tabla 3. Grado de conocimiento sobre bases fisiopatológicas, factores de riesgo y medios de diagnóstico de los profesionales de enfermería del área clínica comparado con el área comunitaria, villavicencio, 2010.

\begin{tabular}{|c|c|c|c|c|c|c|}
\hline \multirow{3}{*}{ Área } & \multicolumn{6}{|c|}{$\begin{array}{l}\text { Grado de conocimiento sobre bases fisiopatológicas, factores } \\
\text { de riesgo y medios de diagnóstico para Diabetes Tipo } 2\end{array}$} \\
\hline & \multicolumn{2}{|c|}{ Alto } & \multicolumn{2}{|c|}{ Regular } & \multicolumn{2}{|c|}{ Bajo } \\
\hline & $\mathrm{n}$ & $\%$ & $\mathrm{n}$ & $\%$ & $\mathrm{n}$ & $\%$ \\
\hline Clínica & 1 & 6.67 & 2 & 13.33 & 12 & 80 \\
\hline Comunitaria & 3 & 20 & 5 & 33.34 & 7 & 46.66 \\
\hline
\end{tabular}

Tabla 4. Grado de conocimiento sobre mantenimiento de control metabólico en la Diabetes Tipo 2 de los profesionales de enfermería del área clínica comparado con el área comunitaria, Villavicencio, 2010.

\begin{tabular}{l|c|c|c|c|c|c}
\hline \multirow{2}{*}{ Área } & \multicolumn{2}{|c|}{ Grado de conocimiento sobre mantenimiento de control metabólico en la Diabetes Tipo 2 } \\
\cline { 2 - 7 } & \multicolumn{2}{|c|}{ Alto } & \multicolumn{2}{c}{ Regular } & \multicolumn{2}{c}{ Bajo } \\
\cline { 2 - 7 } & $\mathbf{n}$ & $\%$ & $\mathbf{n}$ & $\mathbf{n}$ & $\%$ \\
\hline Clínica & 0 & 0 & 1 & 6.67 & 14 & 93.33 \\
Comunitaria & 0 & 0 & 2 & 13.33 & 13 & 86.67 \\
\hline
\end{tabular}

Tabla 5. Grado de conocimiento sobre complicaciones de la Diabetes Tipo 2 de los profesionales de enfermería del área clínica comparado con el área comunitaria, Villavicencio, 2010.

\begin{tabular}{l|c|c|c|c|c|c}
\hline \multirow{2}{*}{ Área } & \multicolumn{3}{|c|}{ Grado de conocimiento sobre complicaciones de la Diabetes Tipo 2 } \\
\cline { 2 - 7 } & \multicolumn{2}{|c|}{ Alto } & \multicolumn{2}{c|}{ Regular } & \multicolumn{2}{c}{ Bajo } \\
\cline { 2 - 7 } & $\mathbf{n}$ & $\%$ & $\mathbf{n}$ & $\%$ & $\mathbf{n}$ & $\%$ \\
\hline Clínica & 0 & 0 & 2 & 13.33 & 13 & 86.67 \\
Comunitaria & 0 & 0 & 1 & 6.67 & 14 & 93.33 \\
\hline
\end{tabular}


Las categorías relacionadas con las limitaciones para el cuidado de enfermería y con las posibilidades y oportunidades para dar educación fueron las siguientes: Recarga laboral y desempeño de funciones no profesionales; ausencia de control del cuidado y delegación de funciones profesionales; y educación como supuesto. Sobre las condiciones para mejorar el cuidado de enfermería ofrecido se identificaron las categorías: Necesidad de educación continuada; y necesidad de trabajo interdisciplinario y apoyo técnico. La descripción de las categorías de análisis identificadas se presenta a continuación.

\section{Recarga laboral y desempeño de funciones no profesionales}

Los profesionales en la mayoría de las instituciones deben cumplir con múltiples actividades, entre otras, las denominadas administrativas, que exigen por ejemplo realizar Ilamadas interinstitucionales para búsqueda de camas, interconsultas, realización de trámites de documentación de los pacientes, etc., que no corresponden al rol del profesional, pero se han ido asumiendo, en la medida en que las instituciones optan por asignarlas. Esta situación deteriora el cuidado que deben brindar los profesionales a las personas hospitalizadas con DM2 y a sus familiares. Ejemplos de enunciados significativos dentro de esta categoría son:

"Por lo menos el sacar fotocopias para pos, no-pos, para solicitudes de autorizaciones, sacar las remisiones, bajarlas, todo el proceso administrativo que hay acá, bajar las remisiones, ir a presentar el paciente, bajar paquetes para autorizaciones, ir a llevar los formatos para que sean autorizados los formatos no-pos cpc, para que sean enviados a los entes territoriales y a las EPSs, todas esas cosas que generalmente son documentos que hay que sacar copias e ir a llevar a diferentes partes nos quitan mucho tiempo."

"Hoy por ejemplo (turno de mañana) no estoy dando medicamentos, hoy estoy solamente administrativo, entonces me toca revisar todos los kárdex de los 60 pacientes que hay, hacer las dietas, revisar las formulaciones de los 60, asignar camas, bueno, todo lo que llegue, los permisos, los pacientes que quieran ingresar alimentos. Todo eso lo hacemos es la jefe administrativa en el día."

\section{Ausencia del control del cuidado y delegación de funciones profesionales}

La recarga laboral en funciones de organización de los servicios o unidades administrativas, impide que los profesionales de enfermería controlen de manera directa el cuidado de enfermería o suministren el cui- dado de enfermería diferente a la administración de medicamentos.

De hecho, las acciones administrativas señaladas anteriormente, obliga a los profesionales a delegar el cuidado de los pacientes al personal auxiliar de enfermería, que por supuesto no siempre está mejor formado para prestar el debido cuidado integral. Incluso, algunos enfermeros reportan que realmente es el personal auxiliar de enfermería el que pasa más tiempo con los pacientes y por lo tanto suministra el cuidado directo de enfermería, a pesar de señalar que es al profesional de enfermería a quien le correspondería orientar y proveer el cuidado, incluyendo el componente educativo para la persona con diabetes. Algunos ejemplos de enunciados significativos que ilustran esta categoría son:

"la persona que está más al pie casi siempre es la auxiliar de enfermería, entonces pues uno le dedica tiempo mientras que va a dar el medicamento".

"...hay veces por ejemplo en mi piso, nos toca dos servicios a la vez una profesional de enfermería. Qué hacemos? Delegar un poconón de funciones en las auxiliares, entonces esa limitación es terrible, terrible para dar educación, no solo al diabético, a todos."

"...todas las actividades que se nos han recargado a enfermería, administrativas y otra serie de cosas de papelería, que no nos deja tener el contacto que quisiéramos tener con el paciente."

\section{Educación como supuesto}

Los profesionales de enfermería en el área clínica, manifiestan que realizan educación durante los contactos que tienen con el paciente hospitalizado, durante los procedimientos de intervención (curaciones, aplicación de medicamentos, otros) por lo que el dedicado a la acción es de tal solo unos minutos. Durante ese tiempo se hace énfasis en el suministro de la información, sin que necesariamente haya una valoración formal de las necesidades individuales, y sin considerar las circunstancias del paciente asociadas al procedimiento que se ejecuta tales como dolor $\mathrm{o}$ ansiedad durante la curación, además se identifica que la información, sin valoración sistemática de necesidades previa es inespecífica por lo que la persona puede no considerarla efectivamente para el mejoramiento de las capacidades de automonitoreo y autocuidado. Algunos ejemplos se esta categoría son:

“...arréglese bien las uñitas, no vuelva a usar más el cortaúñas, una lima de cartón, ande con zapaticos, hay que evitar andar ya en chancletas." 
“..si estoy dando la insulina, entonces le muestro la jeringa de insulina, le muestro el frasquito, le digo su mecanismo de acción, cada cuánto, que por qué se la estoy administrando, qué hace en el paciente." " (a la sesión educativa)...le pongo 10 minutos, no más".

“...uno va con el esquema de Ud. como se lo cuida en su casa, y si estamos en visita, qué más, Ud. como se está alimentando, y si le están haciendo la dieta, o si uno ve que la hemoglobina glicosilada le llegó superalta entonces uno se le acerca y: Hay que ponerle más cuidado a la dieta, o sea unas recomendaciones muy chiquitas, pero sí, sí se hace."

Los profesionales participantes reconocen que la educación no se hace de la forma apropiada ni en la duración requerida e identifican a la limitación de tiempo como una de las causas para esa situación. Algunos enunciados relacionados con esta categoría son:

“...como están organizados los turnos y con todas las actividades que tenemos, no tenemos el tiempo. Que podríamos hacer la posibilidad de sacar un poquito de tiempo, si se puede, pero es demasiada la sobrecarga laboral para hacerlo adecuadamente, con todo los requerimientos y como uno quisiera, no, a mí me parece que no hay el tiempo."

“...a veces la demanda del trabajo de enfermería es mucha y no alcanza el tiempo para hacer muchas actividades. Por lo menos no como uno quisiera darle una educación adecuada a este tipo de pacientes."

\section{Necesidad de educación continuada}

Los profesionales participantes identifican la necesidad de educación continuada como herramienta fundamental para mejorar la calidad de la educación a los pacientes y cuidadores familiares. Se relaciona la educación en salud como una forma importante de prevenir las complicaciones agudas y crónicas que tienen gran impacto en la calidad de vida de las personas.

“... nosotros como personas ser más concientizados y quizás más capacitados en cuanto a los pacientes de diabetes, que no hay que llegar hasta esperar a esa fase final."

“...esto (el servicio) está lleno de diabéticos, pero nosotros no tenemos el cuidado, ni el personal capacitado ni el tiempo disponible para ese tipo de educación."

\section{Necesidad de trabajo interdisciplinario y de apoyo técnico}

Los profesionales de enfermería reconocen la importancia de trabajo en equipo, interdisciplinario, para el adecuado cuidado de las personas con diabetes, reconocen también la necesidad de contar con personal bien entre- nado y dedicado a este tipo de pacientes. Sin embargo, identifican que estos aspectos presentan un déficit notorio en los entornos clínicos en los que laboran. Adicionalmente consideran importante el seguimiento clínico ambulatorio o incluso domiciliario que se le debe ofrecer a las personas con diabetes, particularmente a las personas con escasa escolaridad, ya que requiere de seguimiento estrecho y verificación de aprendizajes. Algunos de los enunciados significativos fueron:

“...lo ideal sería que nosotros no nos limitáramos solo aquí en la institución, sino que nos extendiéramos a la parte ya de su casa, ir a hacerle seguimiento una nutricionista, y el personal de enfermería, cómo hacer las curaciones, cómo ella se administra el medicamento, hacerle un seguimiento."

"...que el paciente salga sabiendo sobre su manejo en casa, yo pienso que eso sería bueno, con que una enfermera estuviera, una sola, nombrada en la institución solamente para dar educación, se lograría mucho."

"si se va y ese señor ni siquiera sabe cómo se aplica (la insulina), qué es lo que tiene que hacer, absolutamente nada, no se le dijo nada de como se tenía que hacer las cosas... cómo se va a mejorar el paciente,... y si eso lo entendiera todo el sistema, sabría que nos sale más caro reconsultar, y esas re-hospitalizaciones donde lo ven tres y cuatro especialistas, que haberle hecho la educación."

Las categorías de análisis que contextualizan la acción educativa en DM2 en el área comunitaria fueron las siguientes:

\section{Educación individualizada y grupal e interdisciplinaria de características variables}

Los profesionales de enfermería participan en educar a las personas con DM Tipo II, en forma individualizada cuando atienden la consulta, con características variables en tiempo de 15-30 minutos y dependiendo del volumen de trabajo. Se orienta sobre la necesidad de controlar dieta, ejercicio, prevención del pie diabético y manejo de los medicamentos básicamente. No se señalan aspectos de intervención para el apoyo emocional, pero son valorados a través de la situación familiar que comunican los pacientes. La educación grupal es interdisciplinaria con nutricionista, médico, terapista física y psicólogo en muchas ocasiones. En algunas de las Instituciones prestadoras del servicio de salud (IPS), la atención es coordinada entre los diferentes profesionales, mejorando la prestación del servicio. En algunas instituciones no siempre existe equipo interdisciplinario suficiente y estable.

"A ellos se les cita cada dos meses a su control médico pero se hace una reunión mensual con el grupo de 
pacientes que quieran asistir, pues no se puede obligar a la parte educativa, no hay mecanismos para la obligatoriedad, se hace una vez al mes y se hace desarrollo de temas de acuerdo a la patología, en este momento se ha hablado de generalidades de pie diabético"

"Acá hay dos tipos de forma; hay un club de crónicos que formaron que son todos los sábados, los reúnen les hacen talleres y actividad física dependiendo de cómo tratarlos y la actividad central de control, tenemos la fortuna que tomamos controles de media hora a diferencia de otras IPS que manejan controles de (20) veinte minutos, eso nos da 10 minutos más para hacer la educación personalizada, dependiendo del paciente se hace la educación". "La que hacen abajo (institución de apoyo) es grupal dependiendo del número de personas que vayan, la que se hace aquí, si es individual dependiendo del control".

\section{Enfermería líder en la coordinación educativa pero insuficiente recurso}

Los profesionales de enfermería suelen ser insuficiente recurso para la atención de los programas de promoción de la salud, ocupan liderazgo en la coordinación educativa, pero están sujetos al tiempo disponible, no siempre suficiente. Por lo que se traduce con la mejor intención, sin programación específica, con participación oportuna o inoportuna. Al mismo tiempo, expresan la necesidad de demostrar su potencial de asistencia profesional, con mejor formación en los aspectos relacionados con la diabetes y la situación de cronicidad que ella plantea.

"La enfermera es la persona que lidera, la calidad de seguimiento, de tamizaje, porque hacemos jornada de tamizaje, tenemos grupo de meta de 18 años en adelante, se hace tamizaje de los elementos que están alterados, es además quien lidera la parte educativa coordina, designa quien dicte el taller o lo dicta ella misma es la que hace el enlace con el equipo de salud y es la que hace la parte de educación, es la que evalúa la adherencia, es la que revisa si el paciente está controlado o no para que se haga la referencia ofrecer un buen cuidado".

"Lo que podemos hacer desde la parte de enfermería es de pronto ser un poco más, no pacíficos si no brindar más bien, con más enfoque la parte educativa, brindar con más cuidado la parte educativa y brindar al paciente la orientación en cuanto al tratamiento y a la medicación, cosa que el internista no hace, que los especialistas no hacen y que el médico general tampoco hace. A uno como enfermero le toca hacer todo".

\section{Necesidad de educación continuada en diabetes}

Los profesionales de enfermería del área comunitaria, frente a las frecuentes complicaciones de los perso- nas con DM2, expresan razonamientos externos a su responsabilidad, que pueden explicar este resultado. De modo similar, algunos profesionales evidencian la necesidad de avanzar con conocimiento, como parte del equipo interdisciplinario para atender la complejidad que exige la atención de las personas con la enfermedad y poder mejorar el cuidado. Los profesionales no hacen mención de haber recibido educación continuada sobre la atención a las personas con diabetes y la importancia de su control y seguimiento.

“...pero pienso que estamos débiles todavía en el complemento de educación al entregar al grupo diabético que ya lo tenemos encuestado y lo tenemos clasificado hay un núcleo como tal a un equipo como tal que va a pertenecer, considero que esa estrategia nos puede fortalecer bastante otra cosa que estamos tratando de buscar es el patrocinio para que una persona se capacite, en Diabetes"

“...por ejemplo ya tenemos el contacto con el doctor Escobar en Bogotá para en la Asociación Diabetológica Colombiana, ellos hacen un curso de capacitación solo de diabetes una semana, pero pues por el costo y la disponibilidad de tiempo ha sido un poquito difícil certificar educadoras auxiliares, estábamos pensando que con la formación del núcleo va hacer mucho más fácil, y que podamos desarrollar el trabajo que exige el curso para graduarnos como educadoras de diabéticos eso es básico, y eso es lo que estamos tratando de conseguir por lo menos para la enfermera que hay acá".

\section{Recursos económicos insuficientes de las instituciones de salud destinados a promoción y prevención}

La expresión de los profesionales de enfermería frente a los recursos requeridos para una excelente prestación del servicio, comúnmente no es satisfactoria. No siempre el recurso humano que debe conformar equipos interdisciplinarios es suficiente, ni está siempre en disponibilidad oportuna, señalándose que se logra una atención en lo posible de la mejor calidad y que necesariamente se deben considerar por las directivas institucionales aspectos para mejorar la prestación del programa específico para la atención a la diabetes, puesto que adicionalmente los recursos materiales también son insuficientes para lograr educación de calidad.

"No en la consulta, solo está asistiendo el paciente diabético, la consulta es individual, es personalizada, no se hace consulta de enfermería, porque por la estructura no tenemos donde meter a la enfermera en un sitio para la consulta". 
"La oportunidad de las consultas no siempre existente, pero se abren alternativas con medicina familiar" "Si esa es la idea mejorar esas oportunidades, pero es complicada esa posibilidad, se podría hacer a nivel administrativo".

\section{Discusión}

Como lo señalan los resultados presentados, los profesionales de enfermería que cuidan a personas con diabetes en el área clínica y en el área comunitaria en Villavicencio tienen limitaciones en el conocimiento sobre diversos aspectos que pueden afectar la calidad del cuidado ofrecido y la seguridad del usuario. El conocimiento tiene mayores limitaciones en los temas relacionados con obtención y mantenimiento de control metabólico y la prevención y el cuidado de las complicaciones, aspectos fundamentales en el cuidado de la persona con diabetes, particularmente en el desarrollo de planes educativos. Las limitaciones reportadas en el conocimiento se presentan en contextos de recarga laboral, delegación de funciones a personal auxiliar, desempeño de función educativa con limitaciones de recursos y tiempo, con grupos interdisciplinarios débilmente constituidos y con escasa oportunidad para formación en servicio o educación continuada. Esta situación debe ser intervenida de manera efectiva con el fin de mejorar las posibilidades de adherencia al tratamiento y prevenir las complicaciones que generan tanto impacto en las esferas sociales y económicas de las personas afectadas y sus familias.

El rol de educador del profesional de enfermería es claro, del mismo modo es evidente la necesidad de poseer información clara y relevante para el desarrollo de las competencias necesarias que permitan conductas sostenidas para el autocontrol y autocuidado (Moser et al., 2009). De hecho, el profesional de enfermería es para el paciente la fuente primaria de información que le permite aplicar en su vida diaria el conocimiento dado, particularmente en el entorno domiciliario y laboral.

En el contexto clínico, se identifica a los enfermeros como el profesional responsable de la educación del paciente lo que incluye el manejo del tratamiento que con frecuencia consiste en la administración de insulina. Sin embargo, se han identificado las limitaciones de los profesionales de enfermería para la toma de decisiones en la administración de insulina teniendo como parámetro una escala de valores ajustable al reporte de glicemia de cada paciente (Savion et al., 2010) lo que impone severas restricciones en el momento de educar a los pacientes sobre el uso de insulina en sus domicilios. Estas restricciones para dar educación se tornan severas en medio de la sobrecarga laboral reportada por los profesionales en el estudio, lo que da como resultado la pérdida de oportunidades de educar aún en hospitalizaciones prolongadas ya que los profesionales no tienen los conocimientos, ni los recursos institucionales para educar en forma apropiada a las personas con DMII que ingresan a las instituciones hospitalarias.

Se ha considerado que la formación de los profesionales de la salud en el tema de la DM2 es de vital importancia para poder acompañar y orientar al enfermo en el desarrollo de habilidades de automonitoreo y autocuidado. Por esta razón la Federación Internacional de Diabetes ha propuesto un plan de formación para profesionales de la salud, incluyendo profesionales de enfermería, en donde se tratan como temas específicos, entre otros, los relacionados con el desarrollo de competencias para autocontrol y autocuidado (International Diabetes Federation, 2011), principales herramientas para la obtención de control metabólico y la prevención de complicaciones. Esta propuesta es concordante con la estrategia global desarrollada por varios años basada en la Educación Terapéutica, proporcionada por el grupo multidisciplinario, generalmente dada a grupos de pacientes de DM2 con aproximación individualizada en varios temas, y valorada con criterios de control metabóli$\mathrm{Co}$, impacto psicosocial, nivel de conocimientos y en menor proporción criterios económicos (Albano et al., 2008).

Adicionalmente, los profesionales de enfermería en el área comunitaria tienen un rol fundamental en la prevención de la enfermedad y la detección precoz de personas de alto riesgo. El conocimiento sobre los diferentes factores de riesgo tales como edad, raza, índice de masa corporal, es importante para identificar tempranamente a las personas a riesgo de desarrollar estados previos a la diabetes. De hecho, se ha mostrado (Zeimer et al., 2008) que los riesgos mencionados podrían ser incluso reemplazados por la determinación de glucosa plasmática casual como pruebas de tamizaje para diabetes o estados prediabéticos, conducta que podría ser implementada en los servicios de salud comunitaria tales como centros de salud y programas de atención a personas de edad adulta.

Las competencias fundamentales de un profesional de enfermería educador incluyen el compromiso de una actitud de aprendizaje y autoformación permanente, participación en elaboración de programas educativos y en la evaluación de los resultados del programa (Kalb, 2008), competencias similares se esperan de 
los profesionales de enfermería educadores en diabetes, quienes deben estar siempre revisando y actualizando sus conocimientos, valorando y evaluando cada caso de persona con diabetes y desarrollando nuevas estrategias para efectivamente integrar el conocimiento significativo a las herramientas del enfermo para adaptarse exitosamente a la enfermedad. De esta manera, la educación continuada se constituye en una herramienta para mantener la calidad de la educación a los pacientes y mantener la calidad del cuidado suministrado. Evidentemente, las instituciones deben comprometerse en el esfuerzo por ofrecer capacitación en servicio y actividades de educación continuada a los profesionales de enfermería y personal de salud involucrado en el cuidado de las personas con DM2.

Sobre este aspecto, la literatura reporta propuestas con diferentes metodologías y vías de acceso tales como cursos vía internet con un abordaje interdisciplinario que han aumentado el nivel de conocimientos de los profesionales de salud en el tema del educación en diabetes (Hall et al., 2007). Opciones similares podrían ser diseñadas y desarrolladas con el fin de responder a las necesidades de conocimientos de los profesionales de enfermería aquí presentadas. Aún en condiciones de limitaciones económicas de las instituciones y de restricciones de recursos, es necesario abordar de manera responsable la formación de los profesionales de enfermería que cuidan a las personas con DM2. Para ello, se pueden diseñar los programas educativos de manera que el tiempo sea optimizado, utilizando diversas metodologías y aprovechando los recursos de aprendizaje que la práctica en servicio ofrece.

Tal es el caso de los programas educativos en servicio y uso del lenguaje estandarizado. Se ha mostrado (Lunney, 2008) cómo los estudios de caso en donde se hace uso de la taxonomía NANDA y los términos de la Clasificación Internacional de Resultados Esperados y de Intervenciones de Enfermería facilita el desarrollo del pensamiento crítico, posibilita la provisión de cuidado de enfermería de calidad, permiten a los profesionales de enfermería familiarizarse con los tipos de pacientes que se encuentran en la práctica clínica o comunitaria, y orientar al personal acerca del cuidado de enfermería de los casos más complejos que impongan retos en los profesionales con escasa experiencia. De manera particular, el entrenamiento en la determinación de los diagnósticos de enfermería señala la importancia de ser consciente de que no se conoce al otro, de que es necesario recabar información sobre el caso específico para poder aproximarse con una información apropiada para cada individuo, lo que a su vez señala la importancia de la comunicación interpersonal para la valoración de necesidades.

La base de la aplicación de las estrategias de valoración parte del principio de que el profesional de enfermería requiere de información sobre las historias de salud y la situación física para obtener los mejores datos que orienten el diagnóstico y por tanto determinen la intervención necesaria para cada persona (Lunney, 2010). Obviamente, estas expectativas no son posibles en instituciones en donde el cuidado directo ha sido delegado casi en su totalidad al personal auxiliar, por lo que el profesional de enfermería no tiene la necesidad ni la oportunidad de comunicarse directamente con el paciente o usuario del servicio con el ánimo de hacer una valoración del individuo y ajustar los planes educativos. Esta circunstancia hace que el cuidado de enfermería sea reemplazado por otras labores diversas relacionadas con el funcionamiento de los servicios y se tienda a pensar en que las cortas comunicaciones esporádicas con los pacientes sean consideradas como sesiones educativas, aún cuando no se tenga ningún conocimiento previo del individuo y no se programen las sesiones con objetivos educacionales, desdibujando con ello la importancia de las intervenciones educativas planeadas y la participación del equipo multidisciplinario en la educación del paciente y familia.

En general, hay un acuerdo global sobre la necesidad de contar con la participación de diversos profesionales de la salud en el desarrollo de las intervenciones educativas tales como profesionales de enfermería, nutricionistas, farmaceutas, médicos, etc. (OPS, 2010). Algunos programas incluyen psicólogos, odontólogos y administradores de negocios, (Valdez et al., 2007) apuntando de manera clara a la importancia de la conformación de grupos interdisciplinarios para el cuidado integral del paciente y para la planeación y desarrollo de las intervenciones de educación.

El conocimiento que poseen los profesionales de enfermería orienta programas de educación a los pacientes con diabetes, lo que a su vez permite obtener control sobre sus acciones de manera que pueden tomar decisiones basadas en información práctica (Fischetti, 2008) evidenciando mejores resultados de control metabólico luego de obtener información detallada sobre dieta, ejercicio y acciones de automonitoreo en niveles de glucosa en sangre. Sin embargo, para que las intervenciones educativas sean apropiadas, es necesario que los profesionales de enfermería puedan suministrar información relacionada con DM2 y su control que responda por lo menos, a las condiciones de edad, género de los enfermos ya que 
hay características específicas de la enfermedad que se pueden presentar de manera diversas dependiendo de la edad del enfermo, tal como sucede en la presentación de signos físicos de hipoglicemia en los adultos mayores (Brown y Abdelhafiz, 2010). Por tal razón, el cuidado de apoyo educativo no podría ser ofrecido sin previa valoración de las variables sociodemográficas básicas, aspecto que tendría que ser reforzado previamente a las intervenciones tanto en el medio clínico como comunitario.

Implementado lo anterior, podría obtenerse un incremento en la expectativa de vida y mejoramiento en las condiciones de su diario vivir a partir de los ajustes positivos en su estilo de vida. Sin embargo, no es ese el único aspecto relevante a mejorar en lo relacionado con los conocimientos de los profesionales de enfermería en diabetes. Las personas con DM2 enfrentan numerosas barreras en su espacio laboral y familiar, tal como lo explora un estudio cualitativo (Thomas, 2011) realizado recientemente que señala que los trabajadores en general minimizan el impacto que la enfermedad puede tener sobre su desempeño laboral a partir de diversos mecanismos cono el de no aplicar el conocimiento adquirido a su propio caso, demostrar optimismo que previene el reconocimiento de los riesgos reales que impone la enfermedad, lo que le permite pensar en los riesgos como un aspecto a futuro sin consecuencias inmediatas, por lo que percibe que no es necesario, al menos de manera rápida, adoptar cambios en estilos de vida. Los profesionales de enfermería deben entonces ser competentes en la identificación por medio de valoración sistemática de este tipo de respuestas en las personas con diabetes a las que ofrecen y en el uso de los canales de interacción con otros profesionales del grupo interdisciplinario con el objeto de ofrecer intervención de manera oportuna. Adicionalmente, los enfermeros deben trabajar continuamente, junto con el equipo interdisciplinario, en la educación de las personas con diabetes para prevenir complicaciones y evitar mortalidad prematura.

Como lo señala Conley (2007), el $40 \%$ de las muertes prematuras en USA se relacionan con estilos de vida inapropiados, por lo que los riesgos globales relacionados con presencia de una enfermedad están asociados a una dieta inadecuada y a actividad física insuficiente. Por tal razón, el conocimiento sobre temas como control metabólico y la obtención de niveles apropiados de glucosa en sangre, es de enorme significancia para la prevención de complicaciones crónicas. De hecho, la propuesta de usar la estrategia de Manejo Intensivo (IM por sus siglas en inglés) está basada en la evidencia de que manteniendo la glu- cosa dentro de los valores normales y aceptando solo el valor normal superior como meta para el control de glicemia en las personas con diabetes será posible evitar la aparición de las temidas complicaciones crónicas que tanto impacto tienen en la calidad de vida de las personas y en su expectativa de vida.

De otro lado, y aún con esta evidencia, se ha mencionado la preocupación por hacer excesivo énfasis en la diabetes y no en la persona, ya que las precisas metas de control, imponen también regímenes estrictos en las personas, lo que pone a prueba sus capacidades para la adaptación rápida a su nueva situación (Fox, 2010). Todos estos elementos deben ser tenidos en cuenta por el profesional de enfermería durante su intervención educativa y de cuidado al enfermo por lo que es necesario que conozca sobre los diversos aspectos de la DM2 y su control y retome el cuidado directo para poder ofrecer a las personas una intervención de calidad que pueda realmente apoyar cambios en los estilos de vida de los enfermos. Claramente, y según lo señalan los hallazgos, los profesionales de enfermería requieren replantear sus conceptos sobre educación en salud y recuperar, con apoyo de las instituciones, su rol de proveedor directo de cuidado a las personas de manera que estas tengan la oportunidad de aprender sobre su autocuidado durante el contacto planeado con los enfermeros.

Estos procesos educativos pueden ser reforzados por medio de la participación de otros actores que se encuentran en el entorno social de la persona con la enfermedad enriqueciendo el ambiente de aprendizaje para los enfermos y sus familias. De hecho, un estudio (Sensenig, 2007) propone la prestación de servicios educativos en base comunitaria a través de servicios de enfermería, apoyado en recurso humano en formación, desarrollando un trabajo colaborativo para ofrecer a las personas con diabetes información individualizada y de calidad a través de las prescripciones de enseñanza que el personal de enfermería establece luego de su valoración y los estudiantes de enfermería desarrollan bajo la supervisión de los profesionales docentes. Además de las sesiones individualizadas de educación, los estudiantes también desarrollan estrategias para evitar la ausencia de los pacientes en las sesiones educativas. Se han publicado también resultados (Strang et al., 2010) de nuevas herramientas y metodologías para la formación de estudiantes de enfermería a nivel de pregrado que ofrecerán cuidado de enfermería a las personas con diabetes en un futuro próximo.

De otro lado, se ha mostrado (Sedó, 2010) que las intervenciones educativas tienen que estar acom- 
pañadas de programas de identificación de barreras percibidas por las personas, antes de poder iniciar los cambios de hábitos no saludables. Las barreras percibidas por las personas con DM2 radican con frecuencia en la dificultad para integrar los múltiples cambios en los estilos de vida y la inclusión de nuevas actividades relacionadas con el automonitoreo, reflejando una limitación en las habilidades de afrontamiento necesarias para obtener control metabólico, Los profesionales de enfermería requieren del conocimiento en temas asociados con afrontamiento, empoderamiento, desarrollo de autoestima y de habilidades para la toma de decisiones. Se ha encontrado que las personas con diabetes, en general, no logran comprender la naturaleza y duración de la enfermedad, tienen dificultades para reconocer que tienen la alteración y que requiere de tratamiento de larga duración, por lo que sus capacidades para adaptación positiva se ven muy limitadas dado que sus creencias y actitudes determinaban la capacidad para obtener control metabólico sostenido. Por lo tanto, se identifica como necesario el reconocimiento y manejo de las barreras percibidas por las personas en forma individual para obtener mejores resultados en el control de la enfermedad (Ali y Jusoff, 2009).

Los programas de intervención diseñados para aumentar la comprensión de la enfermedad por parte de los enfermos y de sus cuidadores y mejorar la adherencia al tratamiento han sido documentados (Jenkins et al., 2010; Lange et al., 2010; Lancheros et al., 2010) y han mostrado la importancia de explorar los recursos comunitarios tales como iglesias, organizaciones de madres, recursos tecnológicos de amplio acceso, etc. que ofrecen una red de apoyo, canalizan información y pueden apoyar el desarrollo de estrategias para mejorar la adaptación a la situación de salud. Este tipo de acciones pueden ser consideradas por los enfermeros de servicios comunitarios de manera que se aumenten las probabilidades de los enfermos de obtener control metabólico y los profesionales de enfermería reciban apoyo necesario considerando las limitaciones de tiempo y recursos humanos reportados.

En los niveles socioeconómicos bajos, las limitaciones educativas impactan seriamente la posibilidad de adquirir la información necesaria para el autocuidado en diabetes. Los profesionales de enfermería que cuidan a estas personas ven, muchas veces con impotencia, como la persona enferma entra y sale de la institución de salud, sin la información necesaria para el control exitoso de la enfermedad. De hecho la información que la persona tiene en ocasiones es tan limitada que su situación podría semejarse a la de una persona inmersa en una cultura diferente con las limitaciones características de esa situación. Los programas de inclusión de las minorías étnicas y población culturalmente diferente de otras latitudes (Ly, 2009; Reinschmidt et al., 2010) podrían ser orientadores para la meta de incluir a todos las personas con diabetes atendidas en las instituciones de salud del nivel local y nacional.

El desarrollo de la conducta empática y sensible a las diferencias socioculturales es una de los elementos de importancia en la relación enfermero-paciente y un ingrediente necesario para el desarrollo de las competencias conductuales que debe adquirir la persona con la enfermedad. La falta de reconocimiento y ajuste a estas diferencias han sido identificadas como elementos significativos que disminuye la adherencia a las recomendaciones de estilos de vida ya que el enfermo percibe al profesional de salud como poco interesado en su bienestar, por lo que el profesional de enfermería debe hacer un esfuerzo extra para entender las diferencias culturales que puedan existir y poder ofrecer un cuidado culturalmente sensible, (Marshall, 2007) que responda a las necesidades reales de las personas y se comunique de manera que sea comprendido y atienda las particularidades culturales de las personas.

Al respecto, se han estudiado comunidades específicas con perfiles culturales muy definidos y se ha encontrado la necesidad de ofrecer información apropiada y educación culturalmente relevante con el fin de que sea involucrada para la adquisición de nuevas habilidades necesarias para el cuidado conveniente de la enfermedad (Washington y Wang-Letzkus, 2009). Esto es particularmente importante en ambientes de recarga laboral, con recursos económicos limitados en donde se debe hacer uso del tiempo de los profesionales para obtener los propósitos educativos y de cuidado previstos.

En conclusión, es necesario ofrecer a los profesionales de enfermería del área clínica y comunitaria de Villavicencio, la oportunidad para cuidar a las personas con DM2 en forma apropiada, atendiendo las recomendaciones globales y nacionales que sobre este tema se han planteado. Existen diferentes propuestas para valorar e intervenir desde el ámbito de enfermería a las personas con DM y esas estrategias pueden ser retomadas, examinadas y aplicadas localmente. Previamente, es necesario desarrollar acciones de carácter institucional que permitan al enfermero el ejercicio profesional del cuidado a las personas, incluyendo las intervenciones educativas, y aportando además los recursos económicos y humanos requeridos para el desarrollo de programas de educación 
continuada a los enfermeros, y el seguimiento y reforzamiento en servicio.

Adicionalmente, las instituciones se deben comprometer en la creación de equipos interdisciplinarios que funcionen de manera coordinada, como elemento fundamental para la oferta de cuidado integral de alta calidad al usuario de los servicios de salud, ofreciendo así intervenciones educativas sensibles a las condiciones socioculturales de las personas con DM2 y que permitan, razonablemente, esperar una disminución de la carga de enfermedad asociada a la presencia de DM2 y a las complicaciones y mortalidad prematura, presentes cuando no fue obtuvo control metabólico sostenido.

\section{Agradecimientos}

Las autoras agradecen a la Universidad de los Llanos por el soporte financiero para el desarrollo de la investigación. Agradece también a los estudiantes principiantes de investigación (EPI) por la participación en el desarrollo del estudio, contribuyendo en la recolección de los datos y realizando un análisis de resultados como requisito de grado para la obtención del título de Enfermera/o. Los estudiantes participantes fueron: William Alberto Garzón Ortiz, Sandra Milena Suarez Henao, Adriana Rivera García y Viviana Juanias Suárez.

\section{Referencias}

Adams K. (2009) Diabetes epidemic: implications for nursing practice. Australian Nursing Journal. Feb 1;16(7): 37. In: ProQuest Nursing \& Allied Health Source [database on the Internet] [cited 2010 Feb 2]. Available from:http://www.proquest.com/; Document ID: 642634901.

Albano MG, Crozet C, D'ivernois JF. (2008) Analysis of the 20042007 literature on therapeutic patient education in diabetes: results and trends. Acta Diabetol 45(4):211-211-9. http:// search.proquest.com/health/docview/195821420/13263FF26 424DAC5F26/55? accountid $=48773$

Ali SM, K. (2009) Barriers to Optimal Control of Type 2 Diabetes in Malaysian Malay Patients. Global Journal of Health Science 1(2):106-106-118. http://search.proquest.com/health/docview/822029617/fulltextPDF/13263FF26424DAC5F26/26?ac countid $=48773$

Asociación Colombiana de Diabetes.(2004) La cartilla de la diabetes. Panamericana Formas e Impresos: Bogotá.

Beto J, Nicholas M. (2009) So Just What Can I Eat? Nutritional Care in Patients with Diabetes Mellitus and Chronic Kidney Disease. Nephrology Nursing Journal. Sep 1;36(5): 497-504; quiz 505. In: ProQuest Nursing \& Allied Health Source [database on the Internet] [cited 2010 Feb 2]. Available from: http:// www.proquest.com/; Document ID: 1884237351.
Bradbury-Jones C. (2009) Globalization and its implications for health care and nursing practice. Nursing Standard. 2009 Feb 25;23(25): 43-7. In: ProQuest Nursing \& Allied Health Source [database on the Internet] [cited 2010 Feb 2]. Available from: http://www.proquest.com/; Document ID: 1659975491.

Brown SHM, Abdelhafiz AH. (2010) Hypoglycemia, intensive glycemic control and diabetes care in care home residents with Type 2 diabetes. Aging Health 6(1):31-31-40. http://search. proquest.com/health/docview/221196383/fulltextPDF/13263 FF26424DAC5F26/32? accountid= 48773

Conley YP, Tinkle MB. (2007) The Future of Genomic Nursing Research. Journal of Nursing Scholarship 2007;39(1):17-17-24. http://search.proquest.com/health/docview/236392002/fullte xtPDF/13263FF26424DAC5F26/27? accountid $=48773$

Fischetti N. (2008) Using Standardized Nursing Languages: A Case Study Exemplar on Management of Diabetes Mellitus. [Consultado 2011 Nov 3] International Journal of Nursing Terminologies and Classifications 19(4):163-163-6. http://search. proquest.com/docview/194431944? accountid=48773

Fox A. (2010) Intensive Diabetes Management: Negotiating Evidence-based Practice. Canadian Journal of Dietetic Practice and Research 71(2):62-62-8. http://search.proquest.com/health/ docview/347840536/fulltextPDF/13263FF26424DAC5F26/5 9 ? accountid $=48773$

García R. (2007) La educación a personas con diabetes mellitus en la atención primaria de salud. Jan. - abril. V.18 n.1. Rev. Cubana. Endocrinol. Instituto nacional de Endocrinología. Ciudad de la Habana. Disponible en: http://scielo.sid.cu/ scielo.php?script=sci_arttext\&lng=pt\&nmr=iso\&ting=pt\&pid =S1561-295320070000100005\#cargo

Hall DL, PharmD., Drab SR, PharmD., Campbell RK, Meyer SM., Smith RB. (2007) A Web-based Interprofessional Diabetes Education Course. Am J Pharm Educ 71(5):1-93. http://search. proquest.com/health/docview/211216859/fulltextPDF/13263 FF26424DAC5F26/52 ?accountid $=48773$

Hicks D. (2009) New role in diabetes care provision. Journal of Diabetes Nursing. July. [consultado 2010 Enero 20] Disponible en: http:/www.highbeam.com/doc/1G1-206593287.html

Hill J. (2009) Reducing the risk of complications associated with diabetes. Nursing Standard. Feb 25;23(25): 49-55; quiz 58. In: ProQuest Nursing \& Allied Health Source [database on the Internet] [cited 2010 Feb 2].Available from: http://www.proquest.com/; Document ID: 1659975501.

International Diabetes Federation. (2011) Position statement: Self-management education. Diabetes self-management education: a right for all. [aprox. 7 pantallas ] 2011 International Diabetes Federation. Disponible en: http://www.idf.org/ node/23502

Jenkins C, Pope C, Magwood G, Vandemark L, Thomas V, Hill K, et al. (2010) Expanding the Chronic Care Framework to Improve Diabetes Management: The REACH Case Study. Progress in Community Health Partnerships 2010;4(1):65-65-79. http:// search.proquest.com/health/docview/845495990/fulltextPDF/ 13263FF26424DAC5F26/51 ?accountid=48773

Kalb KA. (2008) Core Competencies of Nurse Educators: Inspiring EXCELLENCE in Nurse Educator Practice. Nursing Education 
Perspectives 29(4):217-217-9. http://search.proquest.com/ health/docview/236656695/fulltextPDF/13263FF26424DAC5 F26/24? accountid= 48773

Lancheros L, Pava A, Bohórquez A. (2010) Identificación de la adherencia al tratamiento nutricional aplicando el modelo de Conocimientos, Actitudes y Prácticas en un grupo de personas con Diabetes Mellitus tipo 2 atendidas en la Asociación Colombiana de Diabetes. Diaeta [revista en la Internet]. Dic [citado 2011 Nov 21]; 28(133): 17-23. Disponible en: http:// www.scielo.org.ar/scielo.php?script=sci_arttext\&pid=S1852$73372010000400003 \& \operatorname{lng}=\mathrm{es}$.

Lange I, Campos S, Urrutia M, Bustamante C, Alcayaga C, Tellez Á. et al. (2010) Efecto de un modelo de apoyo telefónico en el automanejo y control metabólico de la Diabetes tipo 2, en un Centro de Atención Primaria, Santiago, Chile. Rev. méd. Chile [revista en la Internet]. Jun [citado 2011 Nov 21]; 138(6): 729-737. Disponible en: http://www.scielo.cl/scielo.php?script=sci_ arttext\&pid=S0034-98872010000600010\&Ing=es. doi: $10.4067 /$ S0034-98872010000600010.

Lunney M. (2008) Current Knowledge Related to Intelligence and Thinking With Implications for the Development and Use of Case Studies. International Journal of Nursing Terminologies and Classifications 19(4):158-158-62. http://search.proquest. com/health/docview/194473014/13263FF26424DAC5F26/3 5 ? accountid $=48773$

Lunney M. (2010) Use of Critical Thinking in the Diagnostic Process. International Journal of Nursing Terminologies and Classifications 21(2):82-82-8. http://search.proquest.com/health/ docview/304922117/fulltextPDF/13263FF26424DAC5F26/7? accountid $=48773$

Ly K. (2009) Diabetes: no patients left behind. Community Practitioner 2009;82(2):12-12-3. http://search.proquest.com/health/ docview/213394333/fulltextPDF/13263FF26424DAC5F26/5 0 ? accountid $=48773$

Marshall MC. (2007) Overcoming Barriers to Glycemic Control in African Americans with Type-2 Diabetes: Benefits of Insulin Therapy. J Natl Med Assoc 99(8):868-868-75. http://search. proquest.com/health/docview/214043531/fulltextPDF/13263 FF26424DAC5F26/49? accountid $=48773$

Ministerio de la Protección Social. República de Colombia. (2008). Guía de atención de la diabetes mellitus tipo 2. Disponible en: http://www.nacer.udea.edu.co/pdf/libros/guiamps/guias17.pdf

Moser A, Houtepen R, Spreeuwenberg C, Widdershoven G. (2009) Autonomous Decision Making and Moral capacities. Nurs Ethics 16(2):203-203-18. http://search.proquest.com/health/ docview/201428704/fulltextPDF/13263FF26424DAC5F26/1 2? accountid $=48773$

Organización Mundial de la Salud OMS (2010) [página en internet]. Burden: mortality, morbidity and risk factors. Chapter 1 . En: Global status report on NCDs 2010. WHO. 2010:9-31. Disponible en:http://www.who.int/nmh/publications/ncd_report_chapter1.pdf

Organización Panamericana de la Salud. OPS (2009a) [página en Internet]. Guías ALAD de diagnóstico, control y tratamiento de la Diabetes Mellitus Tipo 2. Presentación de la Dra. Mirta Roses Periago, Directora de la OPS. [consultado en 2010
Enero 23] Disponible en: http://new.paho.org/hq/index. php?option=com_content \&task=view\&id=2164\&ltemid=259

Organización Panamericana de la Salud. OPS (2010) Estrategia de cooperación técnica OPS-OMS. Honduras 2006-2010. Disponible en: http://www.who.int/countryfocus/cooperation strategy/ccs_hnd_es.pdf

Organización Panamericana de la Salud. OPS. (2009b) [página en Internet]. Guías ALAD de diagnóstico, control y tratamiento de la Diabetes Mellitus tipo 2. Versión Interactiva. Panamerican Health Organization. Asociación Latinoamericana de Diabetes. [consultada en 2010 Enero 23] Disponible en: http://new.paho.org/hq/index.php?option=com_content\&task =view\&id=2164\&ltemid $=259$

Population Reference Bureau. PRB (2009). [página de Internet]. Cuadro de datos de la población mundial 2009 [consultado en 2010 Febrero 1] Disponible en: http://www.prb.org/ pdf09/09wpds_sp.pdf

Reinschmidt KM, Teufel-shone N, Bradford G, Drummond RL, Torres E, Redondo F, et al. (2010) Taking a Broad Approach to Public Health Program Adaptation: Adapting a Family-Based Diabetes Education Program. Journal of Primary Prevention 31(1-2):69-69-83. http://search.proquest.com/health/docview /219955466/13263FF26424DAC5F26/40? accountid=48773

Savion I, Khoury K, Alkoken G, Raz I, Leibovitz G, Eldor R, et al. (2010) Glucose Management by Registered Nurses for Adult Patients Hospitalized in Medical Wards: Structured Guidelines (Protocol) and Working Process. Diabetes Spectrum 23(4):268-268-271. http://search.proquest.com/health/docview/816746349/fulltextPDF/13263FF26424DAC5F26/16?a ccountid $=48773$

Sedó P. (2010) Percepciones sobre la diabetes y su padecimiento en personas adultas mayores diabéticas y familiares de un área urbana de Costa Rica. Anales de Gerontología. 6:99-112. Disponible en: http://www.latindex.ucr.ac.cr/geronto-06/geronto-6-07.pdf

Sensenig JA. (2007) Learning Through Teaching: Empowering Students and Culturally Diverse Patients at a Community-Based Nursing Care Center. J Nurs Educ 46(8):373-373-9. http:// search.proquest.com/health/docview/203971280/fulltextPDF/ 13263FF26424DAC5F26/8? accountid $=48773$

Strang SL, Bagnardi M, Williams S. (2010) Tailoring a Diabetes Nursing Elective Course to Millennial Students. J Nurs Educ 49(12):684-684-6. http://search.proquest.com/health/docvie $w / 814808997 / 13263 F F 26424 D A C 5 F 26 / 2$ ?accountid $=48773$

Thomas EA. Diabetes at Work. (2011) AAOHN J 59(5):213-213220.[ Consultado en 2011 Nov. 3] Disponible en: http:// search.proquest.com/docview/864485965/fulltextPDF/132D OFAEB896278C47D $/ 2$ ? accountid $=48773$

Valdez GM, Dadich K A, Boswell C, Cannon S, Irons B, Vickers P, et al. (2007) Planning and Implementing an Interdisciplinary Diabetes Workshop for Healthcare Professionals. The Journal of Continuing Education in Nursing 2007;38(5):232-232-7. http://search.proquest.com/health/docview/223318723/fullte xtPDF/13263FF26424DAC5F26/11 ?accountid=48773

Washington G, Wang-Letzkus M. (2009) Self-Care Practices, Health Beliefs, and Attitudes of Older Diabetic Chinese Americans. J Health Hum Serv Adm 32(3):305-305-23. http://search.pro- 
quest.com/health/docview/199999441/fulltextPDF/13263FF2 6424DAC5F26/28? accountid $=48773$

Weiler DM, Crist JD. (2007) Diabetes Self-Management in the Migrant Latino Population. Hispanic Health Care International 5(1):27-27-33. http://search.proquest.com/health/ docview/205498724/13263FF26424DAC5F26/3?account $\mathrm{id}=48773$
Ziemer DC, Kolm P, Weintraub WS, Vaccarino V, Rhee MK, Caudle JM, et al.(2008) Age, BMI, and Race Are Less Important Than Random Plasma Glucose in Identifying Risk of Glucose Intolerance: The Screening for Impaired Glucose Tolerance Study (SIGT 5). Diabetes Care 31(5):884-884-6. http://search. proquest.com/health/docview/223019547/fulltextPDF/13263 FF26424DAC5F26/23? accountid $=48773$ 